\title{
Effective source mismatch uncertainty evaluation using resistive power splitter up to $18 \mathrm{GHz}$
}

\author{
S.S. Devi ${ }^{2}$, S. Ahmad ${ }^{1, \star}$, K. Patel ${ }^{2}$, P.S. Negi ${ }^{1}$, and V.N. Ojha ${ }^{1}$ \\ 1 CSIR-National Physical Laboratory, New Delhi, India \\ 2 Department of Electronic Science, University of Delhi, South Campus, New Delhi, India
}

Received: 19 June 2015 / Accepted: 7 August 2015

\begin{abstract}
CSIR-National Physical Laboratory (NPL) India is a National Metrology Institute (NMI), which disseminate traceability of the physical parameters in the country. In the field of microwave, it is one of the apex laboratories to provide traceability. In this paper, the method for precession measurement of effective source reflection coefficient using resistive power splitter and mismatch uncertainty evaluation are reported and discussed. Juroshek method is implemented in conjunction with Vector Network Analyzer (VNA) for mismatch uncertainty evaluation by measuring source reflection coefficient from measured S parameters. The measurement results and their associated uncertainty are presented and discussed from $1 \mathrm{MHz}$ to $18 \mathrm{GHz}$ of the resistive power splitter. The complex reflection coefficient of the effective source is determined using indigenously developed automation software. The method adopted is the most convenient way of measuring effective source reflection coefficient whose values are smaller than the manufacturer specs. The mismatch uncertainty has been improved, which is beneficial during the calibration of power sensors along with power meters.
\end{abstract}

Keywords: Resistive power splitter, effective source match, calibration, mismatch uncertainty

\section{Introduction}

The coaxial microcalorimeter along with the thermistor mounts is a primary standard of microwave power, whose traceability is obtained by assigning effective efficiency as a function of frequency [1-4]. The calibration of microwave power sensor using direct comparison technique is to transfer the calibration factor from a primary standard to a reference standard or from a reference standard to a user organization reference standard power sensor. The parameter transfer is through either by direct comparison or calibration one against the other. This is the simplest method to calibrate a power sensor against a reference standard by connecting each in turn to a microwave source, as shown in Figure 1. The accuracy of microwave power measurement depends on the accuracy of power sensor calibration. Implementing direct comparison technique for power sensor calibration by using equation (1), calibration factor of device under calibration $\left(K_{b(D U C)}\right)$ can be evaluated [2]. DUC power sensor has identical reflection coefficient as that of reference power standard, but the reflection coefficient of the source plays an important role in calculating the mismatch factor for calibration using equation (2) and mismatch uncertainty for the power sensor uncertainty evaluation [2]. This paper presents how Juroshek method $[5,6]$ has been used to evaluate the effec-

^ Correspondence: ahmads@mail.nplindia.org tive source reflection coefficient and mismatch uncertainty from $1 \mathrm{MHz}$ to $18 \mathrm{GHz}$ using resistive power splitter. Then by power ratio measurement it transfers the calibration factor, from standard to DUT, to complete the calibration. The signal generator reflection coefficient cannot be neglected in accurate power sensor calibration with small uncertainty. To improve the calibration result and mismatch uncertainty evaluation, we have to perform a mismatch correction, i.e. the complex reflection coefficient of the microwave source has to be evaluated precisely in the calibration model. The complex value is represented in term of real and imaginary components.

$$
K_{b(D U C)}=K_{b(S T D)} \frac{P_{D U C} / P_{r e f}}{P_{S T D} / P_{r e f}} M F
$$

where,

$K_{b(S T D)}$ Calibration factor of the standard

$P_{D U C} \quad$ Power measured by device under calibration

$P_{S T D} \quad$ Power measured by the standard

$P_{\text {ref }} \quad$ Reference power measured

$M F \quad$ Mismatch Factor

where, Mismatch factor is given by:

$$
M F=\frac{\left[1-\Gamma_{D} \Gamma_{G}\right]^{2}}{\left[1-\Gamma_{S} \Gamma_{G}\right]^{2}}
$$




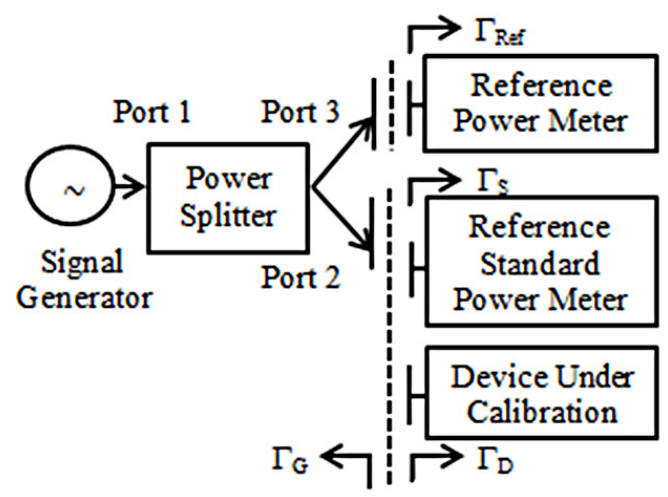

(a)

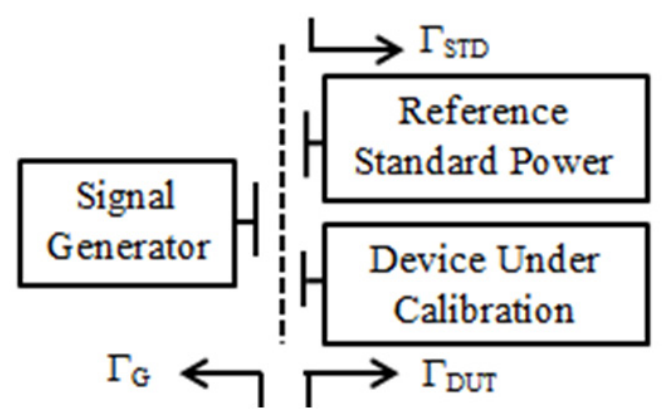

(b)

Fig. 1. (a) Coaxial splitter based power measurement setup. (b) Effective source mismatch.

where, $\Gamma_{G}, \Gamma_{S}$, and $\Gamma_{D}$ are reflection coefficient of signal generator, reference standard and device under calibration respectively.

A resistive power splitter which is a three port device is used in microwave power sensor calibration, where it is important to have a low effective source reflection coefficient over the entire frequency range. However, a significant measurement error can occur due to its directivity, the reflections between the three port of resistive power splitter and the devices connected to it. These errors can be minimized by measuring its equivalent source mismatch. The effective source reflection coefficient at output port 2 of a resistive power splitter may be computed from its complex scattering parameters using equation (3). From this equation we can understand that reflection coefficient of source is basically a function of $\mathrm{S}$ parameters of a three port device and is not affected by the signal generator or detector connected to port 1 and 3 respectively.

$$
\Gamma_{G}=S_{22}-\frac{S_{12} S_{23}}{S_{13}}
$$

where, $S_{i j}$ are complex scattering parameters of a three port resistive power splitter.

One possible way of determining effective source reflection coefficient is using an attenuator at the output port. The system setup is fast, easy and simple. But it may be used only for relatively rough measurement, since the reflection coefficient of a signal generator $\Gamma_{G}$, is more difficult to measure than that of a passive load. Moreover, $\Gamma_{G}$ varies with frequency. For $\Gamma_{G}$ measurement an intermediate component is proposed to use to increase the accuracy. If put an attenuator on the source output, the reflection coefficient of a generator is improved. But low power is a problem if we insert an attenuator. A leveling circuit or ratio measurement can avoid this problem. Resistive power splitter or directional coupler may be used as the intermediate component, as shown in Figure 1a. The effective source mismatch between source and DUC and between source and STD is illustrated in Figure 1b.

The measurement method for evaluating effective source reflection coefficient using three port resistive power splitter proposed by Juroshek is completely based on Vector Network Analyzer measurements [5, 6]. A vector network analyzer is a swept frequency reflectometer system to measure the magnitude and phase angle of reflection and transmission coefficients of a one port or a two port network ( $S$-parameters). The signal transmitted through or reflected from the unknown device is compared in amplitude and phase with a reference signal. The signal separation in the network analyzer is accomplished through the use of directional couplers, bridges and power splitters. The reflection coefficient $\left(\left|S_{11}\right|\right.$ or $\left.\left|S_{22}\right|\right)$ in general $\left(\left|S_{n n}\right|\right)$ of a device is measured in terms of return loss $\left(R L=-20 \log \left(1 /\left|S_{n n}\right|\right)\right.$ against a zero $\mathrm{dB}$ return loss standard whereas the transmission coefficient $\left(\left|S_{12}\right|\right.$ or $\left|S_{21}\right|$ ) of a two port network is measured in terms of attenuation against a zero dB transmission loss standard. The operation of vector network analyzer is based upon the multi-channel tuned IF receiver and the detected IF voltage is proportional to the magnitude of the RF signal.

There are several types of calibrations, defined by ports which are involved and their level of correction is accomplished. These calibration types include namely:

"Full 2-Port": this is the most commonly used and most complete calibration which involves two measurement ports. All four $S$-parameters $\left(S_{11}, S_{12}, S_{21}\right.$, and $\left.S_{22}\right)$ are fully corrected.

"Full 1-Port": We used Full 1-Port calibration in this paper. In this case, a single reflection parameter is fully corrected (either $S_{11}$ or $S_{22}$ ). Both ports can be covered but only reflection measurements will be corrected. This calibration type is useful for reflection-only measurements, including the possibility of doing two reflection-only measurements at the same time. There are a number of imperfections that limit measurements done without calibrations. These imperfections include: match, directivity and frequency response. Through calibration, VNAs provide the highest level of accuracy by correcting for these imperfections, as well as other defects [7-9].

Vector network analyzer has been calibrated using short-open-load-thru (SOLT)/open-short-load (OSL) techniques. Accuracy of the VNA system is verified for reflection and transmission measurement using coaxial precision airlines, $20 \mathrm{~dB}$ and $50 \mathrm{~dB}$ attenuators of the verification kit. To measure reflection parameters $S_{11}$ and $S_{22}$, 


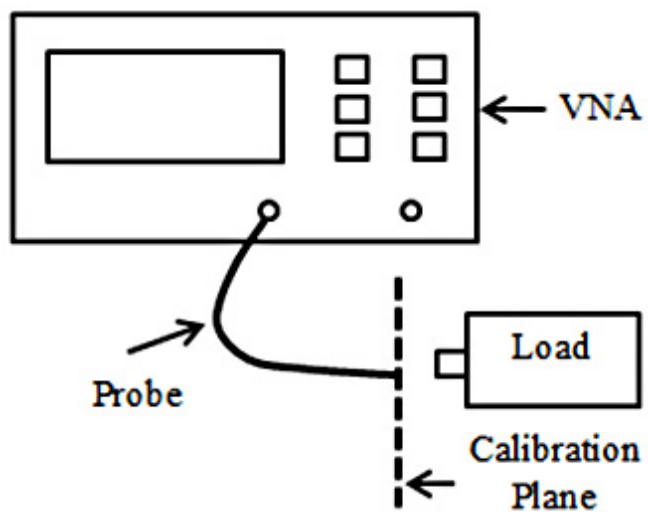

(a)

Fig. 2. (a) VNA One port calibration setup.

a one port device is connected either at test port 1 or at test port 2 of the VNA. The value of $S_{11}$ or $S_{22}$ is the ratio of reference and reflected signals for the two settings of the input switch and the amplitude and phase information of the $S_{11}$ and $S_{22}$ parameters can be read from the display. The observations are repeated atleast five times to compute the type (A) uncertainty. To evaluate the type (B) uncertainty EURAMET guidelines has been followed [10].

\section{Measurement procedure}

Effective source reflection coefficient of a resistive power splitter is determined using VNA and one port calibration technique. In this method a resistive power splitter is connected to an uncalibrated VNA and then performs one port calibration at port 2 of the splitter. The complete measurement process is carried out in two different phases. In first phase, VNA is calibrated for one port calibration. After calibration, three different loads are connected as shown in Figure 2a and their reflection coefficient in real and imaginary form is measured. These measured values would be later used as the known reflection coefficients to solve the matrix equations. In second phase, the resistive power splitter is connected to an uncalibrated VNA as shown in Figure 2b. Port 2 of power splitter is now considered as the effective source port due to the calibration reference plane. Port 1 and 3 of splitter are connected to port 1 and 2 of VNA respectively. One port VNA calibration at the reference plane of the power splitter is repeated again. After performing the calibration, known standards are connected at port 2 of power splitter and reflection coefficient in complex form is measured for each standard [11]. These results are considered as the measured reflection coefficient of known loads. A view of the airline measurement setup for uncertainty evaluation and resistive power splitter setup for $\Gamma_{G}$ evaluation are shown in Figures 3 and 4 respectively.

The known and measured values of load are saved in excel sheet and the indigenously developed automation software is used to calculate the effective source reflec-

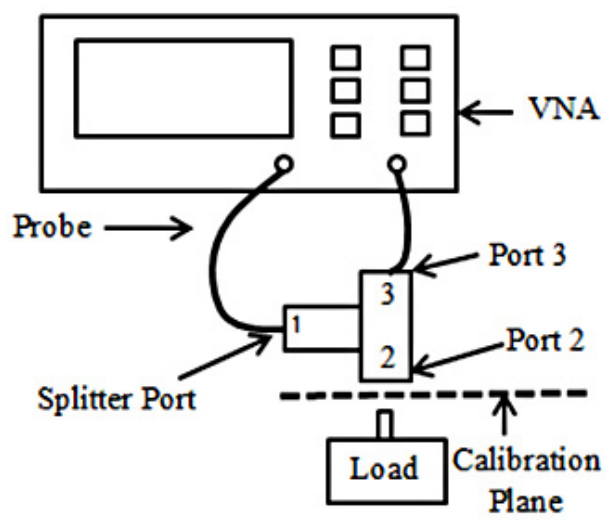

(b)

(b) Measurement setup for $\Gamma_{G}$ evaluation.

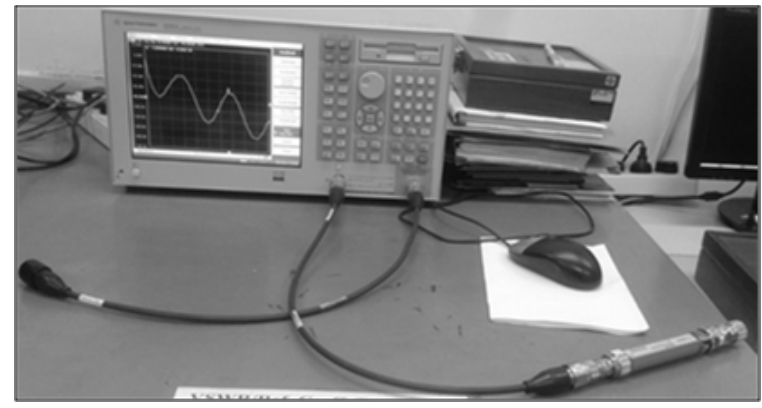

Fig. 3. Airline ripple measurement setup using VNA.

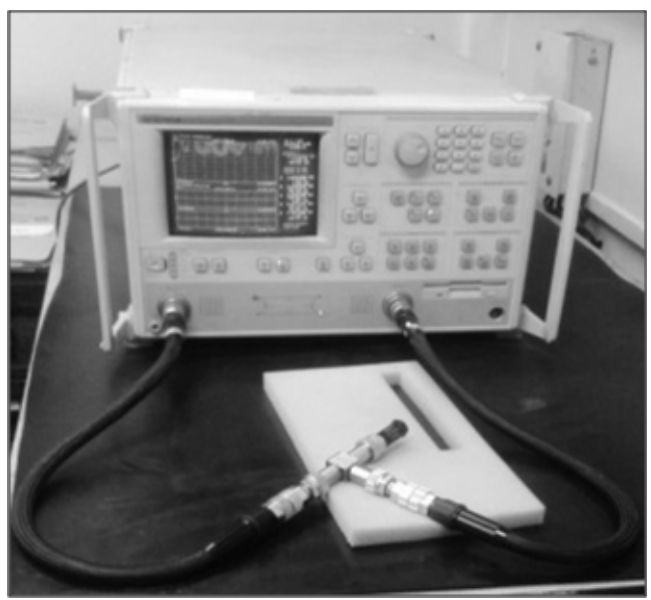

Fig. 4. Pictorial view of the measurement setup.

tion coefficient and it's VSWR. MATLAB platform is used to design and developed automation software for the systematic evaluation of the reflection coefficient $\left(\Gamma_{G}\right)$ and VSWR of the source. A front view of the running software and the flow chart explaining the working of automation software are shown in Figures 5a and 5b respectively. All the measured data has been acquired from VNA and automatically solving complex matrix equations to evaluate effective source reflection coefficient [12]. 


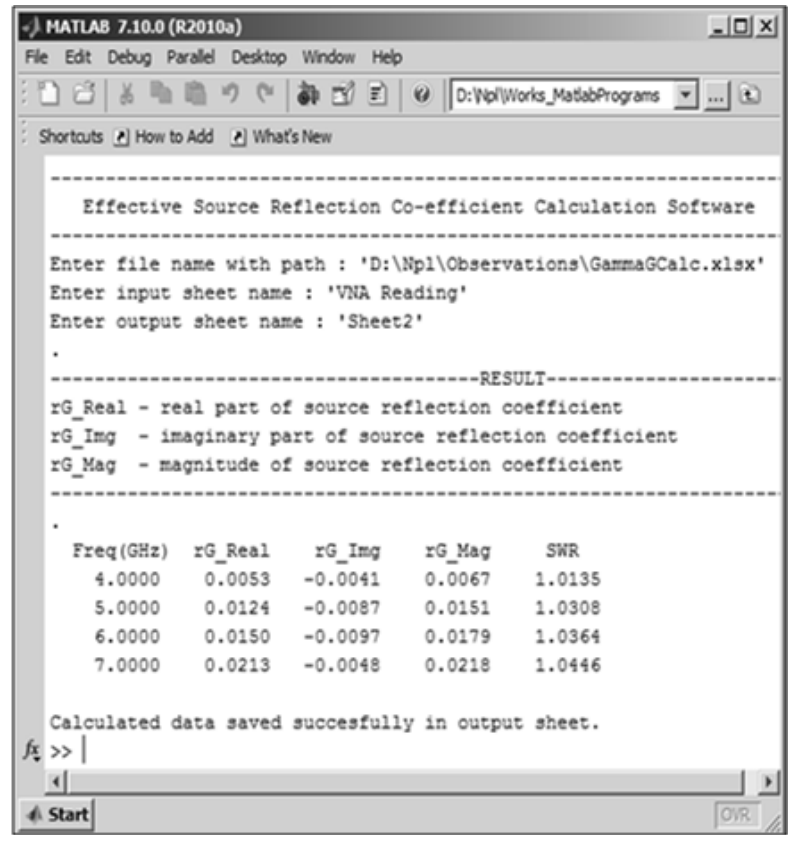

(a)

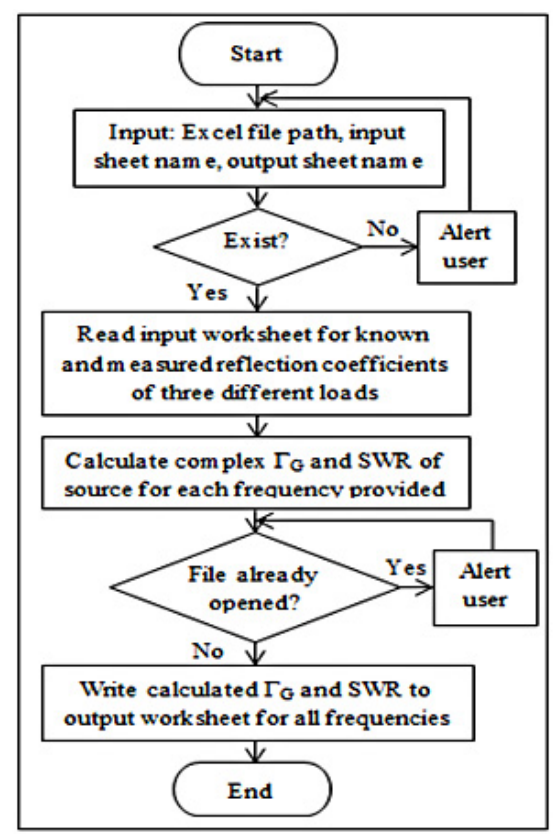

(b)

Fig. 5. (a) View of the automation software. (b) Flow chart for the automation software.

The simplest way of analyzing network problems is to solve the equations for a particular path in signal flow graph [13]. By implementing this method and applying few basics signal flow graph rules the evaluation of complex equations can be greatly simplified. We have used this method to solve and simplify these equations using signal flow graph $[14,15]$. In general $S_{11}$ for DUC using VNA may be given by equation (4), where $\mathrm{a}_{1}$ is incident wave and $b_{1}$ as reflected wave. This method is implemented using VNA, which measures the ratio of incident and reflected voltages at port 2 of resistive power splitter. Using this method, source mismatch can be evaluated if we have three loads of known reflection coefficients and their respective measured reflection coefficient with splitter connected.

$$
\begin{aligned}
& \Gamma=\frac{b_{1}}{a_{1}} \\
& \Gamma=\frac{\Gamma_{M}-e_{00}}{e_{11}\left(\Gamma_{M}-e_{00}\right)+e_{01}}
\end{aligned}
$$

where, $e_{00}$ : directivity, $e_{11}$ : source match, $e_{01} e_{10}$ : reflection tracking.

The quantities directivity, source match and reflection tracking are complex in real and imaginary form that represents imperfection and losses in the resistive power splitter. These three unknown quantities, of the power splitter can be calculated by solving equation (5) with three known loads $[5,6]$. A matrix is formed which can be solved for $e_{11}$ (effective source reflection coefficient).

$$
\begin{aligned}
e_{00}+\Gamma_{S} \Gamma_{M S} e_{11}-\Gamma_{S} \Delta_{E} & =\Gamma_{M S} \\
e_{00}+\Gamma_{O} \Gamma_{M O} e_{11}-\Gamma_{O} \Delta_{E} & =\Gamma_{M O} \\
e_{00}+\Gamma_{L} \Gamma_{M L} e_{11}-\Gamma_{L} \Delta_{E} & =\Gamma_{M L}
\end{aligned}
$$

where $\Delta_{E}=e_{00} e_{11}-e_{01} e_{10}, \Gamma_{x}(x=\mathrm{S}, \mathrm{O}, \mathrm{L})$ are known values for short open and load which is defined by the models of the calibration standards. $\Gamma_{M X}$ is measured value related to wave-quantities for the same. Finally, $e_{11}$ is used as $\Gamma_{G}$ as a reflection coefficient of the source The method described allows fast, accurate and elegant determination of the effective source reflection coefficient of a resistive power splitter [16-18]. The user can thus very easily use $\Gamma_{G}$, e.g. during the calibration of power meters, and can consequently profit from a considerable increase in measurement accuracy. Once the $\Gamma_{G}$ has been measured, it gives an advantage in the calibration of power meter without increasing the DUT measurement time and without incurring additional costs for expensive hardware. Using equations (6)-(8) and solving them for $e_{11}$, which is effective source reflection coefficient $\Gamma_{G}$. We get equation (9) as:

$$
\text { see equation (9) next page. }
$$

\section{Measurement results}

The measurement results for effective source reflection coefficient using equation (9) are shown in Table 1 . The table shows $\Gamma_{G}$ in real and imaginary, its magnitude and VSWR. All the measurement results are obtained by calibrating VNA using calibration kit and verification kit. The effective source reflection coefficient values are determined using resistive power splitter. All the measurements have been carried out in the controlled environmental conditions of temperature $\left(25^{\circ} \mathrm{C} \pm 1{ }^{\circ} \mathrm{C}\right)$ and relative humidity $\mathrm{RH}(50 \% \pm 10 \%)$. 


$$
\Gamma_{G}=\frac{\left(\Gamma_{S}+\Gamma_{O}\right)\left(\Gamma_{M L}-\Gamma_{M O}\right)-\left(\Gamma_{L}+\Gamma_{O}\right)\left(\Gamma_{M S}-\Gamma_{M O}\right)}{\left(\Gamma_{S}+\Gamma_{O}\right)\left(\Gamma_{L} \Gamma_{M L}-\Gamma_{O} \Gamma_{M O}\right)-\left(\Gamma_{L}+\Gamma_{O}\right)\left(\Gamma_{S} \Gamma_{M S}-\Gamma_{O} \Gamma_{M O}\right)}
$$

Table 1. $\Gamma_{G}$ Effective source reflection coefficient.

\begin{tabular}{|c|c|c|c|c|}
\hline \multirow{2}{*}{$\begin{array}{l}\text { Freq } \\
\mathrm{GHz}\end{array}$} & \multicolumn{4}{|c|}{ Effective source reflection coefficient } \\
\hline & Real & Imaginary & Magnitude & VSWR \\
\hline 0.001 & -0.00021 & 0.00027 & 0.0003 & 1.0007 \\
\hline 0.01 & 0.00000 & -0.00012 & 0.0001 & 1.0002 \\
\hline 0.05 & -0.00019 & -0.00033 & 0.0004 & 1.0008 \\
\hline 0.1 & -0.00031 & -0.00054 & 0.0006 & 1.0012 \\
\hline 0.5 & -0.00052 & -0.00009 & 0.0005 & 1.0011 \\
\hline 1.0 & 0.00004 & 0.00012 & 0.0001 & 1.0003 \\
\hline 2.0 & 0.00000 & 0.00034 & 0.0003 & 1.0007 \\
\hline 3.0 & 0.00710 & -0.00860 & 0.0112 & 1.0226 \\
\hline 4.0 & 0.00530 & -0.00410 & 0.0067 & 1.0135 \\
\hline 5.0 & 0.01240 & -0.00870 & 0.0151 & 1.0308 \\
\hline 6.0 & 0.01500 & -0.00970 & 0.0179 & 1.0364 \\
\hline 7.0 & 0.02130 & -0.00480 & 0.0218 & 1.0446 \\
\hline 8.0 & 0.05130 & 0.00410 & 0.0515 & 1.1085 \\
\hline 9.0 & 0.04110 & -0.01900 & 0.0453 & 1.0949 \\
\hline 10.0 & 0.00920 & -0.02030 & 0.0223 & 1.0456 \\
\hline 11.0 & -0.00060 & -0.00370 & 0.0037 & 1.0075 \\
\hline 12.0 & 0.00970 & 0.00090 & 0.0097 & 1.0197 \\
\hline 13.0 & 0.00930 & -0.01780 & 0.0201 & 1.0410 \\
\hline 14.0 & -0.04040 & -0.04750 & 0.0624 & 1.1330 \\
\hline 15.0 & -0.00570 & -0.00670 & 0.0088 & 1.0177 \\
\hline 16.0 & 0.01610 & -0.01690 & 0.0233 & 1.0478 \\
\hline 17.0 & -0.00640 & -0.00560 & 0.0085 & 1.0172 \\
\hline 18.0 & 0.00130 & 0.00100 & 0.0016 & 1.0033 \\
\hline
\end{tabular}

\section{Measurement uncertainty}

Three port resistive power splitter plays an important role in microwave power calibration. Mismatch Uncertainty forms almost half of the total uncertainty budget in power sensor calibration. Therefore the effective source mismatch related to the power splitter has been evaluated. It depends on reflection coefficients of source $\Gamma_{G}$, standard used and device under calibration. The associated mismatch uncertainty has been calculated using the following equation equation (10) [2].

$$
u\left(M U_{x}\right)=\sqrt{ } 2 \Gamma_{G} \Gamma_{x} \quad \text { where, } \quad x=\mathrm{STD}, \mathrm{DUC} .
$$

To calculate mismatch uncertainty, we need VSWR of the microwave source in the entire frequency range. The evaluation is based on VNA measurements using one port calibration technique. The VNA measurement uncertainty has been evaluated based on EURAMET guidelines [10]. This is type (B) uncertainty apart from type (A) uncertainty, which is repeatability. The uncertainty in measuring reflection coefficient of the source depends upon the S parameter measurement of three known loads and is calculated by using the law of propagation of uncertainty [10,19-23]. Using equation (9) we have determined the sensitivity coefficients by evaluating the partial derivative of $\Gamma_{G}$ with respect to all the parameters of the equation. There are six sensitivity coefficients whose values have been evaluated using the following equations.

$$
\begin{gathered}
c_{S}=\frac{\delta \Gamma_{G}}{\delta \Gamma_{S}} \\
c_{O}=\frac{\delta \Gamma_{G}}{\delta \Gamma_{O}} \\
c_{L}=\frac{\delta \Gamma_{G}}{\delta \Gamma_{L}} \\
c_{M S}=\frac{\delta \Gamma_{G}}{\delta \Gamma_{M S}} \\
c_{M O}=\frac{\delta \Gamma_{G}}{\delta \Gamma_{M O}} \\
c_{M L}=\frac{\delta \Gamma_{G}}{\delta \Gamma_{M L}} .
\end{gathered}
$$

Using uncertainties of $\Gamma_{S} \Gamma_{O} \Gamma_{L} \Gamma_{M S} \Gamma_{M O}$ and $\Gamma_{M L}$ and the six sensitivity coefficients evaluated from equation (11)-(16), the combined standard uncertainty $u_{c}\left(\Gamma_{G}\right)$ in effective source reflection coefficient has been determined by equation (17). The results are given in Table 2. The plot of effective source reflection coefficient along with its uncertainty is shown in Figure 6a. The comparison plot of effective source reflection coefficient measured and the manufacturer data is shown in Figure 6b.

$$
\begin{aligned}
& u_{c}\left(\Gamma_{G}\right)=\sqrt{ }\left\{u^{2}\left(\Gamma_{S}\right) c_{S}^{2}+u^{2}\left(\Gamma_{O}\right) c_{O}^{2}+u^{2}\left(\Gamma_{L}\right) c_{L}^{2}\right. \\
& \left.+u^{2}\left(\Gamma_{M S}\right) c_{M S}^{2}+u^{2}\left(\Gamma_{M O}\right) c_{M O}^{2}+u^{2}\left(\Gamma_{M L}\right) c_{M L}^{2}\right\}
\end{aligned}
$$

\section{Discussion}

The method for measuring effective source mismatch using resistive power splitter has been demonstrated. Source reflection coefficient $\left(\Gamma_{G}\right)$ measurement has been done from $1 \mathrm{MHz}$ to $18 \mathrm{GHz}$ using type $\mathrm{N}$ resistive power splitter. The measurement process uses conventional one port VNA calibration technique to determine $\Gamma_{G}$ at port 2 of the splitter. The minimum and maximum difference between measured values of $\Gamma_{G}$ using this method and manufacturer specifications are 0.0076 at $14 \mathrm{GHz}$ and 0.0684 
Table 2. Effective source reflection coefficient and uncertainty.

\begin{tabular}{ccc}
\hline Freq. & $\begin{array}{r}\text { Effective source reflection coefficient } \\
\text { with uncertainty } \\
\text { GHz }\end{array}$ & $\begin{array}{c}\text { Source (VRC) } \\
\text { Uncertainty }\end{array}$ \\
\hline 0.001 & 0.0003 & \pm 0.0057 \\
\hline 0.01 & 0.0001 & \pm 0.0056 \\
\hline 0.05 & 0.0004 & \pm 0.0057 \\
\hline 0.1 & 0.0006 & \pm 0.0058 \\
\hline 0.5 & 0.0005 & \pm 0.0055 \\
\hline 1.0 & 0.0001 & \pm 0.0059 \\
\hline 2.0 & 0.0003 & \pm 0.0064 \\
\hline 3.0 & 0.0112 & \pm 0.0184 \\
\hline 4.0 & 0.0067 & \pm 0.0184 \\
\hline 5.0 & 0.0151 & \pm 0.0186 \\
\hline 6.0 & 0.0179 & \pm 0.0185 \\
\hline 7.0 & 0.0218 & \pm 0.0183 \\
\hline 8.0 & 0.0515 & \pm 0.0187 \\
\hline 9.0 & 0.0453 & \pm 0.0227 \\
\hline 10.0 & 0.0223 & \pm 0.0184 \\
\hline 11.0 & 0.0037 & \pm 0.0184 \\
\hline 12.0 & 0.0097 & \pm 0.0185 \\
\hline 13.0 & 0.0201 & \pm 0.0185 \\
\hline 14.0 & 0.0624 & \pm 0.0178 \\
\hline 15.0 & 0.0088 & \pm 0.0183 \\
\hline 16.0 & 0.0233 & \pm 0.0182 \\
\hline 17.0 & 0.0085 & \pm 0.0182 \\
\hline 18.0 & 0.0016 & \pm 0.0250 \\
\hline & &
\end{tabular}

at $18 \mathrm{GHz}$ respectively. This method is simple, convenient and gives accurate measurement results. Though, all the measurement values lie within the manufacturer specifications but the results are better than the given specifications. The effective source reflection coefficient $\left(\Gamma_{G}\right)$ and its uncertainty $\delta\left(\Gamma_{G}\right)$ are plotted w.r.t. frequency as shown in Figure 6a. We have observed that the uncertainty in measurement of $\left(\Gamma_{G}\right)$ increases with respect to frequency.

The significance of knowing the precise value of $\Gamma_{G}$ using resistive power splitter may be appreciated by considering a typical power sensor calibration situation. Suppose we need to assign calibration factor to a power sensor at $18 \mathrm{GHz}$ that has a reflection coefficient of 0.019 . Using the measurement setup shown in Figure $2 \mathrm{~b}$ and equation (1) calibration factor may be assigned to a power sensor. To estimate mismatch uncertainty between RF source and DUC, one has to know the reflection coefficients of DUC and RF Source. DUC reflection coefficient can be measured using VNA and the mismatch uncertainty directly depends upon the value of effective source reflection coefficient $\left(\Gamma_{G}\right)$. To compare the results at $18 \mathrm{GHz}$ we use manufacturer's data $\left(\Gamma_{G}=0.0700\right)$ and the measured data $\left(\Gamma_{G}=0.0016\right)$. Using equation (9), mismatch uncertainties between $\mathrm{RF}$ source and power sensor are evaluated as $1.88 \mathrm{E}-03$ and $4.30 \mathrm{E}-05$ respectively. Hence, it may be concluded that the effective source

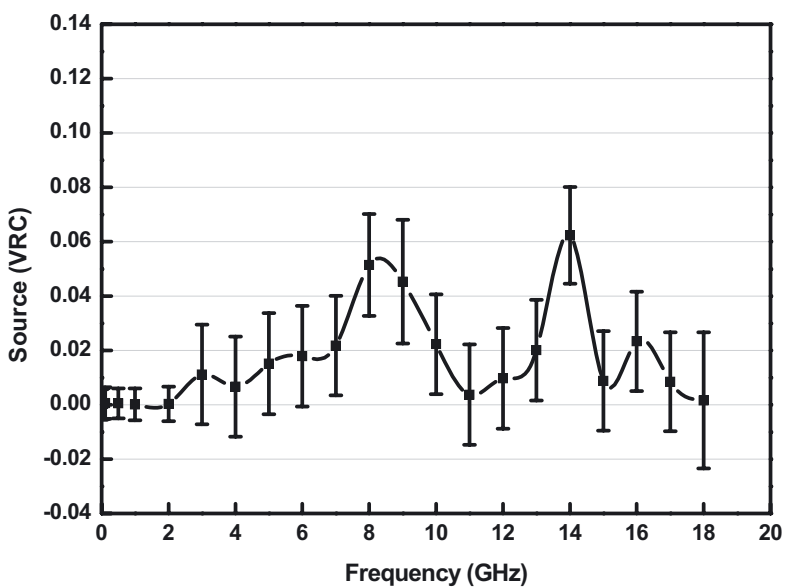

(a)

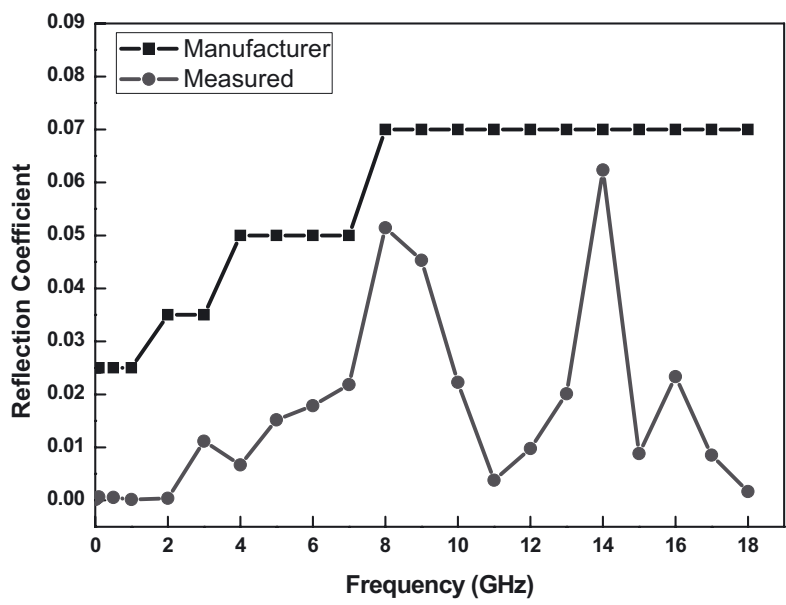

(b)

Fig. 6. (a) Plot of $\Gamma_{G}$ with uncertainty as a function of frequency. (b) Plot to compare $\Gamma_{G}$ measured and manufacturer data.

reflection coefficient $\left(\Gamma_{G}\right)$ measured improves the corresponding mismatch uncertainty, which will further improved the overall combined standard uncertainty of the power sensor at $18 \mathrm{GHz}$. The effective source reflection coefficient $\left(\Gamma_{G}\right)$ measured by implementing the above mentioned method and given by the manufacturer are plotted w.r.t. frequency as shown in Figure 6b.

\section{Conclusion}

We have evaluated the value of $\Gamma_{G}$ effective source reflection coefficient using resistive power splitter together with its uncertainty evaluation by solving the derived equations for three unknowns that are used to calibrate the single port of VNA. Automation software has been developed to implement this method for effective source reflection coefficient measurements. Measurement of $\Gamma_{G}$ using $\mathrm{N}$ type resistive power splitter has been done from $1 \mathrm{MHz}$ to $18 \mathrm{GHz}$. For the estimation of uncertainty, EURAMET guidelines have been followed and the associated uncertainty has been evaluated for all the frequencies. 
This method results in the improvement of power sensor calibration uncertainty. The mismatch uncertainty by measuring the effective source reflection coefficient plays an important role in evaluating the uncertainty for power sensor calibration, which further improves the power sensor calibration uncertainty. This paper has discussed the method to evaluate the reflection coefficient of the source using resistive power splitter and its associated uncertainty. The minimum and maximum difference between measured values of $\Gamma_{G}$ using this method and manufacturer specifications are 0.0076 at $14 \mathrm{GHz}$ and 0.0684 at $18 \mathrm{GHz}$ respectively. Hence, by using this method there is an improvement in mismatch uncertainty, which will further improved the overall combined standard uncertainty. The repeatability of the measurements again confirms the same improvement in the mismatch uncertainty. Hence, by implementing this method we are able to determine equivalent source mismatch, which is an important part of estimating power sensor calibration uncertainty.

Acknowledgements. The authors would like to thank Director, NPL-India for his encouragement and motivation for this work. We would like to thank Mr. Bijendra Pal for his valuable discussion. Furthermore, we would appreciatively acknowledge the continued support of Ms Archana Sahu and Ms Jyoti Chauhan from Microwave Standards for their help in VNA measurements.

\section{References}

1. A. Fantom, Radio frequency and microwave power measurement, IEE Electrical Measurement Series 7, London, UK, Peregrinus, 1990

2. Y. Shan, X. Cui, RF and Microwave Power Sensor Calibration by Direct Comparison Transfer 1. National Metrology Centre, A*STAR, 2. National Institute of Metrology, 1. Singapore, P.R. China

3. S. Ahmad, P.S. Negi, Coaxial Microcalorimeter - National standard for Microwave Power upto $18 \mathrm{GHz}$ at NPLI, Indian J. Pure Appl. Phys. 52, 170-174 (2014)

4. N.S Chung, J. Shin, H. Bayer, R. Honigbaum, Coaxial and waveguide microcalorimeters for RF and MW power standard, IEEE Trans. Instrum. Meas. IM-30, 2, 460-464 (1989)

5. J.R. Juroshek, A direct calibration method for measuring equivalent source mismatch, Microw. J. 40, 106-118 (1997)

6. Measurement method for determining the equivalent reflection coefficient of directional couplers and power splitters, Application note, Rohde and Schwarz

7. D. Rytting, Improved RF hardware and calibration methods for network analyzers, in Proc. of the RF and Microwave Measurement Symposium, Hewlett-Packard Co., Santa Rosa, CA, 1991
8. K. Patel, P.S. Negi, P.C. Kothari, Complex S-parameter measurement and its uncertainty evaluation on a vector network analyzer, Measurement, 42, 145-149 (2009)

9. K. Patel, P.S. Negi, Estimation of complex and linear uncertainties in S-parameter measurements for metrology applications, Int. J. Electromagn. Appl. 2, 85-104 (2012)

10. European Association of National Metrology Institutes, Guidelines on the evaluation of vector network analysers (VNA), EURAMET cg-12 Version 2.0 (03/2011)

11. J. Tippet, R. Speciale, A rigorous technique for measuring the scattering matrix of a multiport device with a 2-port network analyzer, IEEE Trans. Microwave Theory Techniques, MIT-30, 661-686 (1982)

12. A. Saood, V.K. Rustagi, R. Aggarwal, B. Pal, Development of an automated data acquisition system for absolute determination of effective efficiency of RF power mounts using coaxial microcalorimeter, MAPAN - J. Metrol. Soc. India, 22, 63-68 (2007)

13. J.C. Tippet, R.A. Speciale, A rigorous technique for measuring the scattering matrix of a multiport device with a two-port network analyzer, IEEE Trans. Microwave Theory and Techniques, 30, 661-686 (1982)

14. H. Patterson, Signal-flow graphs simplify microwave circuit analysis, Microwave and RF (1995) 99-108

15. G.L. Madonna, Simple technique for measuring source reflection coefficient while characterizing active devices, IEEE Trans. Microwave Theory and Techniques 50, 564-569 (2002)

16. R.C. Powell, Determination of the reflection correction when using a symmetrical two-resistor power splitter to calibrate a power sensor, IEEE Trans. Instrum. Measur. 36, (1987)

17. P. Persson, An algorithm for the evaluation of the residual directivity ripple trace, ANAMET, Middlesex, U.K., Tech. Rep. ANAMET Report 034, 2002

18. R.D. Moyer, Techniques for measuring the effective source reflection coefficient of two-resistor power splitters, IEEE Trans. Instrum. Meas. 36, 23-28 (1987)

19. B. Hall, M. Rodriguez, The uncertainty of the direct method for measuring the equivalent source mismatch of a power splitter: A case study in using software for uncertainty calculation, ANAMET, Middlesex, UK, Tech. Rep. 45 (2004)

20. Ojha V.N., Evaluation and expression of uncertainty in measurement, MAPAN - J. Metrol. Soc. India 13, 71-84 (1998)

21. Guidelines for estimation and expression of uncertainty in measurement results, NABL Document No. 141, Department of Science and Technology, Govt. of India, New Delhi, 2000

22. N.M. Ridler, M.J. Salter, An approach to the treatment of uncertainty in complex S-parameter measurements, Metrolgia 39, 295-302 (2002)

23. K. Yhland, J. Stenarson, Measurement uncertainty in power splitter effective source match, IEEE Trans. Instrum. Measur. 56 (2007) 